\title{
CASOS DE FLEBITE NOTIFICADOS EM SISTEMA DE INFORMAÇÃO DE UM HOSPITAL UNIVERSITÁRIO
}

\section{Karla Hellen Dias Soares', Adrian Thaís Cardoso Santos Gomes da Silva², Bárbara Maria Lopes da Silva Brandão ${ }^{3}$, Adélia Cristina Monteiro Pereira ${ }^{4}$, Estela Maria Leite Meirelles Monteiro ${ }^{5}$ and Roseane Lins Vasconcelos Gomes ${ }^{6}$}

${ }^{1}$ Mestranda do Programa de Pós-graduação em Enfermagem da Universidade Federal de Pernambuco, Recife (PE), Brasil; 2Enfermeira egressa da Universidade Federal de Pernambuco, Recife (PE), Brasil; ${ }^{3}$ Residente do Programa de Residência Multiprofissional em Saúde da Família da Universidade Federal de Pernambuco, Recife (PE), Brasil; ${ }^{4}$ Mestre pelo Programa de Pós-graduação em Enfermagem e Enfermeira do Hospital das Clínicas da Universidade Federal de Pernambuco, Recife (PE), Brasil; 5Docente do Programa de Pós-graduação em Enfermagem e Programa de Pós-graduação em Saúde da Criança e do Adolescente da Universidade Federal de Pernambuco, Recife (PE), Brasil; ${ }^{6}$ Docente do Departamento de Enfermagem da Universidade Federal de Pernambuco, Recife (PE), Brasil

\section{ARTICLE INFO}

\section{Article History:}

Received $11^{\text {th }}$ March, 2020

Received in revised form

$26^{\text {th }}$ April, 2020

Accepted $08^{\text {th }}$ May, 2020

Published online $25^{\text {th }}$ June, 2020

\section{Key words:}

Segurança do Paciente, Flebite, Sistemas de Informação, Cuidados de Enfermagem

*Corresponding author: Karla Hellen Dias Soares

\begin{abstract}
Este estudo objetivou caracterizar os casos de flebite notificados em sistema de informação de um hospital universitário. Estudo quantitativo, exploratório, realizado em um hospital escola no estado de Pernambuco, Brasil. A amostra foi composta de 69 notificações de casos de flebite referentes ao período de 2014 a 2018. Os dados foram coletados no mês de março de 2019 através do software hospitalar de vigilância à saúde. Abordaram-se variáveis sociodemográficas e clínicas do paciente, relacionadas ao incidente de flebite e informações do notificador. Os dados foram analisados por meio de estatística descritiva com o uso do programa Statistical Package for the Social Sciences (SPSS), versão 18. Os casos de flebite notificados predominaram no sexo feminino $(50,7 \%)$, com ocorrência durante internação hospitalar $(98,6 \%)$, em enfermaria de cirurgia geral $(40,9 \%)$. Entre as possíveis causas registradas, as mais frequentes foram antibioticoterapia $(34,3 \%)$ e fragilidade capilar $(28,6 \%)$. Concluiu-se que a maior ocorrência dos casos de flebite se deu em mulheres, em situação de internação, com hipótese etiológica secundária ao tratamento e condição clínica vascular. Os achados reforçam a importância de adoção de boas práticas preventivas e do uso de sistemas informatizados para gestão de risco e segurança do paciente.
\end{abstract}

Copyright (C) 2020, Beathriz Silva Santiago et al. This is an open access article distributed under the Creative Commons Attribution License, which permits unrestricted use, distribution, and reproduction in any medium, provided the original work is properly cited.

Citation: Karla Hellen Dias Soares, Adrian Thaís Cardoso Santos Gomes da Silva, Bárbara Maria Lopes da Silva Brandão et al. "Casos de flebite notificados em sistema de informação de um hospital universitário”, International Journal of Development Research, 10, (06), 36552-36555.

\section{INTRODUCTION}

Eventos adversos são definidos como erros ou incidentes que resultam em dano desnecessário ao paciente e tem sua ocorrência diretamente relacionada a um conjunto de falhas, tais como práticas inadequadas, conduta profissional errônea ou despreparada e estrutura de trabalho inapropriada (Siman et al., 2017). Estes eventos ganham destaque ao gerar inúmeros impactos, que vão desde as consequências econômicas, abrangendo os gastos com internações, medicamentos e procedimentos diagnósticos, até os custos sociais que envolvem absenteísmo e aumento da morbimortalidade (Duarte et al., 2015). Na década de 90, a exposição do relatório americano To Err is Human do Institute of Medicine (IOM), revelou elevada incidência de eventos adversos, em sua maioria ocasionada pelo erro humano, em diversos hospitais dos Estados Unidos (EUA). Estima-se que 44 a 98 mil cidadãos americanos morrem, por ano, devido a erros na assistência à saúde (Institute of Medicine, 2000). Fatores relacionados à deficiência no trabalho e organização da equipe de enfermagem têm sido apontados como contribuintes para a 
maior ocorrência de eventos adversos. Entre eles, destacam-se a falha na execução da sistematização da assistência de enfermagem (SAE), erro na distribuição de tarefas, muitas vezes sem a supervisão necessária, negligência e intensa sobrecarga de trabalho. A enfermagem tem papel importante não apenas na execução de medidas de prevenção e auxílio ao tratamento, mas também na notificação dos incidentes (Mascarenhas et al., 2019). Dentre os eventos adversos possíveis tem-se a flebite. Esta se caracteriza como complicação localizada ao redor da inserção do cateter, e surge como um processo inflamatório desenvolvido na camada interna da parede vascular (Barbosa et al., 2016). A Infusion Nurses Society (INS) estima que a taxa de ocorrência de flebite deve ser 5\% em toda população, entretanto, países como Reino Unido e Irã tem apresentado índices acima de 50\%. A INS aponta que tal fenômeno pode se apresentar em quatro graus distintos: Grau 1 - presença de eritema podendo ou não haver dor; Grau 2 - eritema, dor e/ou edema; Grau 3 - quando além dos sinais referidos há o endurecimento e presença de cordão venoso palpável; Grau 4 - nos casos em que, aditivamente ao grau 3, ocorre formação de cordão venoso longo maior que $2,54 \mathrm{~cm}$, com secreção purulenta local (Infusion Nurses Society, 2011). A equipe de enfermagem exerce importante atuação nas medidas de prevenção de eventos adversos relacionados à flebite e vigilância à saúde.

O acompanhamento de Enfermagem contínuo, desde a admissão até o momento da alta, influencia na qualidade das ações de segurança do paciente (Braga et al., 2018; Souza et al., 2015). Associado à assistência de Enfermagem, o emprego da notificação constitui instrumento imprescindível no monitoramento adequado de risco assistencial e na prevenção de danos à saúde. $\mathrm{O}$ uso de sistemas informatizados pode ampliar e agilizar o processo de registro e de acesso às notificações, otimizando a resolução de incidentes no ambiente hospitalar. A gestão da informação em tempo real por meio do sistema auxilia no planejamento e execução de ações para controle de casos e proteção qualificada do paciente (Ministério da Educação, 2016). A prevenção de danos relacionados ao cuidado torna-se um desafio para os profissionais e instituições de saúde, sendo importante a celeridade na identificação precoce dos eventos adversos, incluindo a ocorrência de casos de flebite. $\mathrm{O}$ reconhecimento e análise destes casos poderá oportunizar uma gestão eficiente da assistência e o estabelecimento de uma rede de procedimentos padronizados para oferta de cuidados seguros. Diante do exposto, este estudo objetivou caracterizar os casos de flebite notificados em sistema de informação de um hospital universitário.

\section{MATERIAIS E MÉTODOS}

Trata-se de uma pesquisa quantitativa, com abordagem exploratória, realizada em um hospital universitário no estado de Pernambuco. A população de estudo se constituiu da totalidade dos casos de flebite notificados $(n=69)$. Foram utilizados dados secundários oriundos de registro eletrônico, através do software de vigilância em saúde e gestão de riscos assistenciais hospitalares, VIGIHOSP. Foram considerados elegíveis ao estudo os casos de flebite notificados em sistema de informação no período de 2014, ano de implantação do VIGIHOSP, à 2018. Por se tratar de amostragem censitária, não se aplicou critérios de inclusão e exclusão. A coleta de dados ocorreu no mês de março de 2019 na Unidade de Gestão de Riscos Assistenciais.
Utilizou-se como instrumento de coleta o formulário de notificação do sistema VIGIHOSP, o qual aborda variáveis sociodemográficas do paciente (sexo, idade, raça/cor), variáveis relacionadas ao local de atendimento (internação, ambulatório, urgência/emergência), ao incidente de flebite (identificação, período, material utilizado, relação com a medicação em uso, identificação prévia do risco, consequência do evento adverso e possíveis causas) e às informações do notificador (local de trabalho, função/formação). Os dados presentes nos formulários de notificação foram tabulados em planilha eletrônica desenvolvida no software Microsoft Office Excel versão 2007. Posteriormente, foi realizada a análise descritiva através do programa estatístico Statistical Package for the Social Sciences (SPSS), versão 18. As variáveis quantitativas foram apresentadas a partir de medidas de tendência central e de medidas de dispersão. Já as variáveis categóricas foram apresentadas por meio de frequências absoluta e relativa. A pesquisa foi aprovada pelo Comitê de Ética em Pesquisa do Hospital das Clínicas da UFPE/Ebserh, sob protocolo 3.142.745 e número CAAE 04776818.4.0000.8807. A obtenção das informações ocorreu mediante a assinatura do Termo de Compromisso para Utilização de Dados pelo pesquisador responsável. O estudo foi isento da utilização de Termo de Consentimento Livre e Esclarecido em virtude do tipo de coleta, sendo todos os dados coletados a partir das informações constantes no sistema informatizado da instituição.

\section{RESULTADOS}

Foram analisados dados de 69 pacientes com caso notificado de flebite, dos quais a maioria era do sexo feminino $(50,7 \%$, $n=35,0)$, de cor parda $(62,4 \%, n=43,0)$, com média de idade de 52,51 anos $( \pm 19,78)$, variando entre 1 a 83 anos. Com relação ao ano da notificação, $85,5 \%(n=59,0)$ dos casos de flebite foram notificados no ano de 2018 , sendo $14,5 \%(n=10,0)$ dos casos notificados no ano de 2017. As veias acometidas no incidente foram principalmente, mediana cubital $(23,4 \%$, $\mathrm{n}=16,0)$, cefálica $(21,7 \%, \mathrm{n}=15,0)$, do arco dorsal da mão $(21,7 \%, \mathrm{n}=15,0)$, basílica $(14,5 \%, \mathrm{n}=10,0)$, mediana $(10,1 \%$, $\mathrm{n}=7,0)$ e cefálica acessória $(1,4 \%, \mathrm{n}=1,0)$. Em $7,2 \%$ dos casos notificados não houve a identificação da veia acometida. $\mathrm{O}$ material mais usado na punção venosa foi o abocath $(84,1 \%$, $\mathrm{n}=58,0)$, seguido do cateter íntima $(7,2 \%, \mathrm{n}=5,0)$ e microfusor $(2,9 \%, n=2,9)$. Em 5,8\% $(n=4)$, foram utilizados outros materiais não especificados. Em 50,7\% $(n=35,0)$ dos casos de flebite, os notificadores afirmaram que o evento adverso não apresentava relação com o uso de medicamento. Entretanto, dos $34(49,3 \%)$ casos que poderiam ter relação, 61,8\% $(n=21,0)$ apresentaram contingência associada ao uso de antimicrobiano. Em 37,7\% $(n=26,0)$ dos casos notificados não ocorreu identificação prévia do risco de flebite. No entanto, na maioria das notificações $(62,3 \%, n=43,0)$ houve identificação antecipada do risco pelo profissional. Quanto ao impacto e consequências associadas à flebite, identificou-se que em $92,8 \%(n=64,0)$ os casos foram classificados como não grave, em $4,3 \%(n=3,0)$ a hospitalização foi prolongada e em $2,9 \%$ $(n=2,0)$ o incidente provocou incapacidade temporária. $\mathrm{Na}$ apreciação das possíveis causas que levaram ao referido incidente foi computado que, dos 69 casos de flebite notificados, 34 apresentaram causa desconhecida (49,3\%), enquanto $35(50,7 \%)$ apresentaram sete possíveis causas para a ocorrência de flebite, sendo a antibioticoterapia (34,3\%, $\mathrm{n}=12,0)$ a mais citada, seguida da fragilidade capilar $(28,6 \%$, $\mathrm{n}=10,0)$, condição clínica do paciente $(22,9 \%, \mathrm{n}=8,0)$, técnica 
inadequada de inserção do cateter intravenoso periférico (CIP) $(14,3 \%, n=5,0)$, propriedades farmacológicas $(11,4 \%, n=4,0)$, tempo de permanência do cateter $(5,7 \%, \mathrm{n}=2,0)$ e incompatibilidade entre fármacos $(2,9 \%, \mathrm{n}=1,0)$. A maior frequência de notificações ocorreu com pacientes em internação hospitalar $(98,6 \%, n=68)$, apenas $1,4 \%(n=1,0)$ dos pacientes notificados com incidente de flebite eram oriundos do ambulatório. Quanto ao tempo para ocorrência do evento adverso, a maior parte dos casos de flebite aconteceram em até sete dias de internamento $(43,9 \%, n=29,0)$. Quanto à unidade de assistência dos casos de flebite registrados, as notificações foram mais frequentes nas enfermarias de cirurgia geral $(40,9 \%)$ e de doenças infecto parasitárias $(11,6 \%)$, e menos frequentes na enfermaria de clínica pediátrica $(1,4 \%)$, centro obstétrico $(1,4 \%)$ e ambulatório de oncologia $(1,4 \%)$. O profissional que mais notificou os eventos adversos classificados como flebite foi o enfermeiro (97,2\%). Os residentes e os estudantes da área de saúde apresentaram baixo número de notificações $(1,4 \%)$.

\section{DISCUSSÃO}

Estudos demonstram maior frequência de flebite no gênero feminino, estando esse fenômeno relacionado tanto à elevada circulação hormonal que predetermina a camada íntima a processos inflamatórios, como também, à anatomia das veias periféricas, visto que os vasos são mais ínfimos e difíceis de serem visualizados (Nobre e Martins, 2018; Beccaria et al., 2018). Contudo, outro estudo aponta o predomínio do acometimento de flebite em homens (Beccaria et al., 2018). Em relação à faixa etária,a maior frequência de casos de flebite em adultos difere de outros estudos que evidenciam maior número de casos em pacientes idosos. Pesquisas apontam que idade acima de 65 anos constitui fator de risco para ocorrência deste evento, uma vez que, os idosos apresentam maior fragilidade na rede vascular e camada íntima das veias, com maior predisposição a inflamações e aumento da chance de desenvolvimento de flebite (Buzatto et al., 2016). A maior proporção de casos de flebite em pacientes com cor de pele autodeclarada parda corroborou com os resultados de estudo realizado em um hospital de ensino localizado em Rio Branco - AC. (Enes et al., 2016). Embora outro estudo tenha comprovado significância estatística entre a cor de pele referida e o grau de flebite, não foram encontrados argumentos que justificassem tal associação (Urbanetto et al., 2016).

Quanto às características clínicas da flebite, há controvérsias na literatura no que se refere à relação entre o desenvolvimento de flebite e a região anatômica (Urbanetto et al., 2016; Buzatto et al., 2016). As veias cubitais, apesar de apresentarem maior calibre, retrataram número de flebite superior quando comparadas às veias da região da mão (Buzatto et al., 2016). Sabe-se que a escolha do local da punção ocorre de acordo com a disponibilidade de vasos sanguíneos do paciente, entretanto, é preferível que a mesma seja realizada nos pontos distais dos membros superiores. A inserção de dispositivos intravenosos deve ser evitada em regiões articulares, visto que são locais com maior propensão à perda do vaso por obstrução ou infiltrações ocasionadas por lesões e/ou perfuração. Isto explica a maior frequência de flebites na fossa cubital (Alves et al., 2018). Tendo em vista que a antibioticoterapia foi registrada como provável causa nos casos de flebite, é possível justificar essa ocorrência pelo $\mathrm{pH}$ de algumas medicações, que quanto mais ácido, maior o risco de flebite química (Urbanetto et al., 2017). Corroborando esse fato, um estudo em Porto
Alegre com 171 pacientes retratou que indivíduos em uso de antibióticos apresentavam elevadas taxas de flebite (Urbanetto et al., 2016). No tocante ao surgimento de flebite e tempo de permanência do cateter, o Center for Disease Control and Prevention (CDC) recomenda que, em adultos, o dispositivo intravenoso não ultrapasse uma estadia superior entre 72 a 96 horas para minimizar os riscos de infecção e flebite (Alves et al., 2018). Pesquisa realizada na Turquia demonstrou que os pacientes que apresentaram tempo de permanência do acesso venoso entre 49 e 72 horas, obtiveram maior ocorrência de flebite (Atay et al., 2018). Contudo, um estudo exploratório não identificou resultados significantes ao comparar o tempo de permanência dos dispositivos com o desenvolvimento do referido evento adverso (Enes et al., 2016). No que se refere à ocorrência de flebite secundária à condição clínica do paciente, a presente pesquisa traz resultados semelhantes a outros estudos, que evidenciaram maior frequência deste evento em pacientes portadores de doenças crônicas e infecciosas (Enes et al., 2016). Isso pode ser esclarecido pelo fator imunológico do paciente e tempo de internação, o qual influencia no risco de infecções, a exemplo de um estudo realizado com pacientes pediátricos hospitalizados (Suliman et al., 2020).

As notificações do evento adverso efetuaram-se em diversos setores da instituição, o que mostra a ocorrência de casos de flebite registrados em pacientes desde o serviço de ambulatório, enfermarias até a unidade de terapia intensiva. A enfermaria de cirurgia geral foi o setor hospitalar que apresentou quase metade das notificações, em consonância com estudo realizado no Paraná-Brasil, que demonstrou que a maior parte dos pacientes acometidos se encontrava na unidade de clínica médica e cirurgia geral (Souza et al., 2015). O registro de ocorrência de flebite de pacientes em tratamento ambulatorial pode estar relacionado a ampliação de procedimentos invasivos ocorridos com pacientes nesta modalidade de assistência, como serviço de quimioterapia, bloco cirúrgico de procedimentos com menor complexidade, entre outros. A ocorrência de flebite nesta situação também tende a apresentar uma subnotificação, sendo necessário estratégias de orientação e acompanhamento dos pacientes. A notificação, quando realizada de maneira correta, permite observar as características do caso, como também as possíveis causas e consequências para o desfecho do evento. Os sistemas de notificação são ferramentas imprescindíveis para garantir a segurança do paciente no cenário intra-hospitalar. Por meio destes sistemas é possível conhecer e identificar os possíveis eventos adversos decorrentes da assistência à saúde (Ferezin et al., 2017) e direcionar medidas voltadas à prevenção, controle e redução de casos de flebite (Schmitt et al., 2016). Além disso, requer considerar que o direcionamento das ações de segurança do paciente, mediante planejamento para evitar e reduzir possíveis danos, resguarda o paciente, mas também a equipe de saúde.

\section{Conclusão}

A pesquisa em questão revelou que, quase a totalidade das notificações dos casos de flebite foram realizadas por enfermeiros, o que indica conhecimento da existência da ferramenta informatizada por parte da categoria. Entretanto, notou-se que as notificações muitas vezes foram registradas de maneira incompleta, com ausência de informações essenciais para o esclarecimento do objeto de estudo. A ausência ou falhas na notificação dos casos de flebite impossibilitam a elucidação de possíveis falhas e o planejamento de medidas 
voltadas à prevenção, controle e redução desta ocorrência. Destarte, reconhecer a relevância das notificações corretas dos incidentes a partir do uso de sistemas informatizados pela gestão de riscos assistenciais e segurança do paciente, para promover um maior engajamento e sensibilização dos profissionais de saúde na notificação voluntária dos casos de flebite, para adoção de boas práticas preventivas para redução deste evento adverso. A presente investigação apresentou como limitação a ausência de informação ou imprecisão em alguns dos registros de notificação, situação que prejudicou a realização de uma investigação mais profunda e consistente sobre o fenômeno estudado. Emerge salientar que a subnotificação e a disponibilização de um recorte temporal ainda sucinto, também constituíram fatores limitantes na apreensão dos achados.

\section{REFERÊNCIAS}

Alves, J. L., Rodrigues, C. M., \& Antunes, A. V. 2018. Prevalência de flebite em uma unidade de internação clínica de um hospital universitário brasileiro de alta complexidade. Revista Brasileira de Ciências da Saúde, 22(3):231-236. Diponível em: https://doi.org/10.22478/ufpb.2317-6032.2018v22n3.27078

Atay, S., Sen, S., \& Cukurlu D. 2018. Phlebitis-related Peripheral Venous Catheterization and the Associated Risk Factors. Nigerian Journal of Clinical Practice, 21(7):827831. Disponível em: https://doi.org/10.4103/ njcp.njcp_337_17

Barbosa, A. K. C., Carvalho, K. R. C., \& Moreira, I. C. C. C. 2016. Phlebitisoccurrence in venousaccess. Enfermagem em Foco, 7(2):37-41. Disponível em: https://doi.org/ 10.21675/2357-707X.2016.v7.n2.792

Beccaria, L. M., Contrin, L. M., Werneck, A. L., Machado, B. D., Sanches, E.B. 2018. Incidence of phlebitis in adult patients. Revista de Enfermagem UFPE on line, 12(3):745752. Disponível em: https://doi.org/10.5205/1981-8963v12i3a230454p745-752-2018

Braga, L. M., Parreira, P. M., Oliveira, A. S. S., Mónico, L. S. M., Arreguy-Sena, C., \& Henriques, M. A. 2018. Phlebitis and infiltration: vascular trauma associated with the peripheral venous catheter. Revista Latino-Americana de Enfermagem, 26:e3002. Disponível em: https://doi.org/ 10.1590/1518-8345.2377.3002

Buzatto, L. L., Massa, G. P., Peterlini, M. A. S., Whitaker, I. Y. 2016. Factors associated with phlebitis in elderly patients with amiodarone intravenous infusion Acta Paulista de Enfermagem, 29(3):260-266. Disponível em: http://dx.doi.org/10.1590/1982-0194201600037

Duarte, S. C. M., Stipp, M. A. C., Silva, M. M., \& Oliveira, F. T. 2015. Adverse events and safety in nursing care. Revista Brasileira de Enfermagem, 68(1):144-154. Disponível em: https://doi.org/10.1590/0034-7167.2015680120p

Enes, S. M. S., Opitz, S. P., Faro, A. R. M. C., \& Pedreira, M. L. G. 2016. Phlebitis associated with peripheral intravenous catheters in adults admitted to hospital in the Western Brazilian Amazon. Revista da Escola de Enfermagem da USP, 50(2):263-271. Disponível em: https://doi.org/10.1590/S0080-623420160000200012
Ferezin, T. P. M., Ramos, D., Caldana, G., Gabriel, C. S., \& Bernardes A. (2017). Analysis of adverse event reporting at accredited hospitals. Cogitare Enfermagem, 22(2):e49644. Disponível em: http://dx.doi.org/10.5380/ce.v22i2.49644.

Infusion Nurses Society. 2011. Infusion nursing Standards of pratice. Norwood, MA: Infusion Nursing Society, 34 (1 Supl):S1-92. Disponível em: http://incativ.es/ documentos/guias/INS_Standards_of_Practice_2011[1].pdf

Institute of Medicine. 2000. To err is human: building a safer health system. Washington, DC: The National Academy Press. Disponível em: https://doi.org/10.17226/9728

Mascarenhas, F. A. S., Anders, J. C., Gelbcke, F. L., Lanzoni, G. M. M., \&Ilha P. 2019. Facilities and difficulties of health professionals regarding the adverse event reporting process. Texto \& Contexto Enfermagem, 28:e20180040. Disponível em: http://dx.doi.org/10.1590/1980-265X-TCE2018-0040

Ministério da Educação. 2016. Diretriz EBSERH para ações em segurança do paciente e vigilância em saúde: manual básico. Brasília, DF:EBSERH. Disponível em: https://www.saude.rj.gov.br/comum/code/MostrarArquivo. php? $\mathrm{C}=\mathrm{Njc} 5 \mathrm{Mw} \% 2 \mathrm{C} \% 2 \mathrm{C}$

Nobre, A. S. P., \& Martins, M. D. S. 2018. Prevalence of peripheral intravenous catheter-related phlebitis: associated factors. Revista de Enfermagem Referência, SerIV(16):127-138. Disponível em: http://dx.doi.org/ 10.12707/RIV17058

Schmitt, M., Haddad, M. C., Rossaneis, M., Pissinati, P. S., \&Vannuchi, M.T. 2016). Examination of notifications of technical defects in technical surveillance at a public university hospital. Vigilância sanitária em debate, 4(3):3541. Disponível em: https://doi.org/10.22239/2317269x.00750

Siman, A. G., Cunha, S. G. S., \& Brito, M. J. M. 2017. The practice of reporting adverse events in a teaching hospital. Revista da Escola de Enfermagem da USP, 51:e03243. Disponível em: http://dx.doi.org/10.1590/S1980220X2016045503243

Souza, A. E. B. R., Oliveira, J. L. C., Dias, D. C., \& Nicola, A. L. 2015. Prevalence of phlebitis in adult patients admitted to a university hospital. Revista da Rede de Enfermagem do Nordeste, 16(1):114-122. Disponível em: https://doi.org/10.15253/2175-6783.2015000100015

Suliman, M., Saleh, W., Al-shiekh Hind, Taan, W., \&AlBashtawy, M. 2020. The incidence of peripheral intravenous catheter phlebitis and risk factors among pediatric patients. Journal of Pediatric Nursing, 50:89-93. Disponível em: https://doi.org/10.1016/j.pedn.2019.11.006

Urbanetto, J. S., Muniz, F. O. M., Silva, R. M., Freitas, A. P. C., Oliveira, A. P. R., Santos, J. C. R. 2017. Incidence of phlebitis and post-infusion phlebitis in hospitalised adults. Revista Gaúcha de Enfermagem, 38(2):e58793. Disponível em: http://dx.doi.org/10.1590/1983- 1447.2017.02.58793

Urbanetto, J. S., Peixoto, C. G., \& May, T. A. 2016. Incidence of phlebitis associated with the use of peripheral IV catheter and following catheter removal. Revista LatinoAmericana de Enfermagem, 24:e2746. Disponível em: https://doi.org/10.1590/1518-8345.0604.2746 